\title{
THE DEPTH FIRST PROCESSES OF GALTON-WATSON TREES CONVERGE TO THE SAME BROWNIAN EXCURSION
}

\author{
By JeAn-François Marckert And Abdelkader MokKadem \\ Université de Versailles St-Quentin
}

\begin{abstract}
In this paper, we show a strong relation between the depth first processes associated to Galton-Watson trees with finite variance, conditioned by the total progeny: the depth first walk, the depth first queue process, the height process; a consequence is that these processes (suitably normalized) converge to the same Brownian excursion. This provides an alternative proof of Aldous' one of the convergence of the depth first walk to the Brownian excursion which does not use the existence of a limit tree. The methods that we introduce allow one to compute some functionals of trees or discrete excursions; for example, we compute the limit law of the process of the height of nodes with a given out-degree, and the process of the height of nodes, root of a given subtree.
\end{abstract}

1. Introduction. In this paper, we study some depth first processes associated to Galton-Watson trees conditioned by their total progeny. Consider $\xi$, a nonnegative, integer-valued random variable that satisfies:

$$
\begin{aligned}
& \mathbb{E}(\xi)=1, \quad 0<\operatorname{Var}(\xi)=\sigma^{2}<+\infty, \\
& \text { there exists a constant } \alpha>0 \text { s.t. } \mathbb{E}\left(e^{\alpha \xi}\right)<+\infty .
\end{aligned}
$$

We consider a Galton-Watson branching process with offspring $\xi$, starting with 1 individual in generation 0 . We write $\tau$ for the family tree of this branching process and $\Omega$ the probability space of all trees with the law induced by $\xi$. We note $\Omega_{n}$ the space of size $n$ trees endowed by the conditional law given $|\tau|=n$.

The depth first search. Let $\tau$ be an ordered tree with $n$ nodes. We define a function [see Aldous (1991), page 260]

$$
\tilde{f}:\{0, \ldots, 2 n-2\} \rightarrow \text { nodes of } \tau\},
$$

which we regard as a walk around $\tau$, as follows:

$$
\tilde{f}(0)=\text { root. }
$$

Given $\tilde{f}(i)=v$, choose, if possible, the left-most child $w$ of $v$ which has not already been visited, and set $\tilde{f}(i+1)=w$. If not possible, let $\tilde{f}(i+1)$ be the parent of $v$.

Received February 2001; revised March 2002.

AMS 2000 subject classifications. 05C05, 60F99, 60G50, 60J80.

Key words and phrases. Galton-Watson trees, simple tree, depth, Brownian excursion, subtree, ladder variable, moderate deviations. 


\subsection{The processes.}

The depth first walk $(D F W)$. For a tree $\tau \in \Omega_{n}$, we call the DFW of $\tau$, the process $V_{n}$ defined by

$$
V_{n}(i)=d(\operatorname{root}, \tilde{f}(i)), \quad 0 \leq i \leq 2 n-2 .
$$

For $i$ from 0 to $n-1$, let $v_{i}$ be the $i$ th new node visited by the depth first procedure on $\tau \in \Omega_{n}\left(v_{0}=\right.$ root $)$ and

$$
\xi_{i}=\text { the outdegree of } v_{i}=\text { the number of children of } v_{i} .
$$

The depth first queue process $(D F Q P)$. For a tree $\tau \in \Omega_{n}$, the DFQP, $S_{n}$ is defined by $S_{n}(0)=0$ and

$$
S_{n}(j)=\sum_{i=0}^{j-1}\left(\xi_{i}-1\right) \quad \text { for any } 1 \leq j \leq n .
$$

The height process. The height process of $\tau \in \Omega_{n}, h_{n}$, is defined by

$$
h_{n}(i)=d\left(\operatorname{root}, v_{i}\right) \quad \text { for } 0 \leq i \leq n-1 .
$$

Note that the DFW, the DFQP as well as the height process characterize the associated tree.

The height of nodes with a given out-degree. Consider $j$ such that $p_{j} \neq 0$; for a tree $\tau \in \Omega_{n}$, the process $h_{n}^{(j)}$ is defined by $h_{n}^{(j)}(0)=0$ and

$$
h_{n}^{(j)}(m)=d\left(\operatorname{root}, \tilde{v}_{m}\right) \quad \text { for } m \geq 1
$$

where $\tilde{v}_{m}$ is the $m$ th node with out-degree $j$ in the list $\left(v_{0}, v_{1}, \ldots, v_{n-1}\right)$. If such a node does not exist, set $h_{n}^{(j)}(m)=0$.
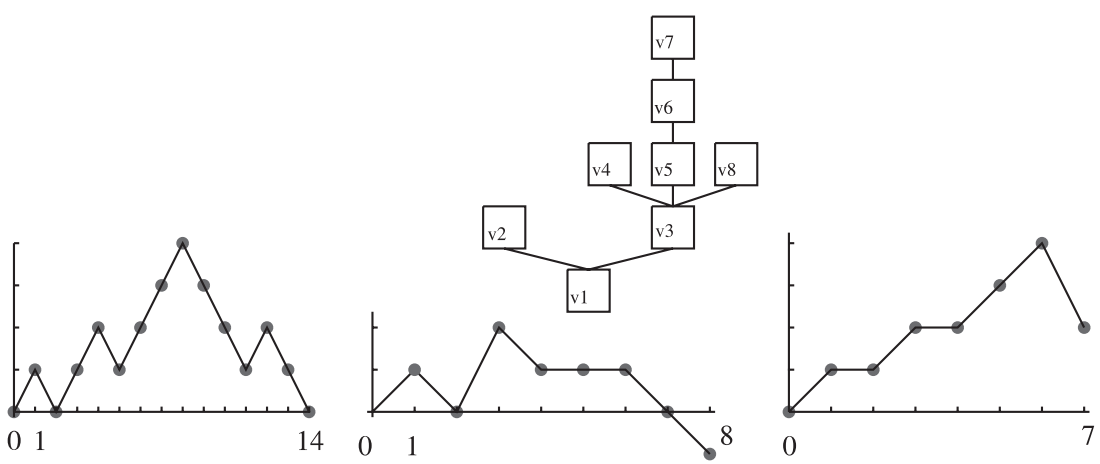

FIG. 1. A tree and its associated DFW, DFQP and height process. 
The height of nodes, root of a given type subtree. Let $u$ denote a fixed tree in $\Omega$ with $|u|=\lambda$ and such that

$$
\mathbb{P}(u)>0 .
$$

For a tree $\tau$ from $\Omega_{n}$, the process $h_{n}^{u}$ is defined by $h_{n}^{u}(0)=0$ and

$$
h_{n}^{u}(m)=d\left(\operatorname{root}, \bar{v}_{m}\right) \quad \text { for } m \geq 1
$$

where $\bar{v}_{m}$ is the $m$ th node in the list $\left(v_{0}, v_{1}, \ldots, v_{n-1}\right)$, root in $\tau$ of a subtree equal to $u$; if no such node exists, set $h_{n}^{u}(m)=0$.

1.2. Comments and previous works. Our paper is cast in term of a critical Galton-Watson process. The simply generated trees in the sense of Meir and Moon (1978) (binary trees, general order trees, Cayley trees, Schröder trees, etc.), can be seen as conditioned critical Galton-Watson trees with an ad hoc offspring distribution [see Aldous (1991) or Drmota and Gittenberger (1997) for a translation index].

As noted by Kennedy (1976), the distribution on $\Omega_{n}$ is unchanged if we replace $\xi$ with another progeny $\xi^{\prime}$, in the same exponential family. Then, there is no loss of generality in considering critical branching process.

Our aim is to show strong relations between the five depth processes presented above; a consequence of these relations is that these five processes (suitably normalized) converge to the same Brownian excursion.

Lately, a lot of works have been published on the properties of depth search processes as well as on breadth first search processes. One can cite, for example, the works of Aldous (1991, 1993, 1998), Drmota and Gittenberger (1997), Takàcs (1993) and Chassaing, Marckert and Yor (2000).

In recent works, Le Gall (2000), Le Gall and Le Jan (1998) and Duquesne and Le Gall (2002) investigated the asymptotic distribution of the height process of a sequence of independent Galton-Watson trees; in these works, the offspring variance can be infinite. The limit height process obtained is a Lévy process, which is the reflected Brownian motion, when the variance is finite. One can also mention the works on LIFO queue of Limic (2000, 2001) who uses the connection between queueing models and branching processes, particularly between the queue lenght and the height process.

The limit of $V_{n}$ is known; in his famous paper Continuum random tree III, Aldous (1993) has shown that the DFW, suitably normalized converges to the normalized standard excursion. Here are the main lines of his proof: he first shows that the Galton-Watson tree conditioned by its total progeny converges (suitably normalized) to a limit object: the continuum random tree. Next, he proves that the distribution of the continuum random tree is unique; it does not depend on the law of the progeny (up to a change of scale). He uses then the fact that for the 
geometrical offspring distribution, the limit of the DFW is the Brownian excursion; thus, it is also the limit of the DFW for the other families of trees.

In the present paper, we assume that $\xi$ has exponential moments, though Aldous assumes only the existence of the variance. The exponential moment assumption is necessary to obtain exponential concentration type inequalities in the theorems. We come back on this difference in the conclusion.

We think that the new approach presented in the present paper has several interests.

- The proofs given here are simple and just rely on well-known parts of probability theory: the ladder height and ladder epochs of simple random walk, the law of large number and concentration inequalities.

- This new approach provides (by Theorem 2) a strong relation between the DFQP and the DFW (illustrated by the figures at the end of the paper). This relation between these two processes seems to be unknown. Using large or moderate deviations on DFQP which are rather easy to obtain (DFQP is an usual discrete excursion), one can obtain (upper bounds on) large or moderate deviations on some functionals on the tree: the height, the mean of the depth of the nodes, ....

- The methods used are sufficiently general to provide concentration inequalities for the uniform distance between the five processes described in the beginning of the paper.

- The unicity of the continuum random tree: Theorem 1 below shows that the DFW converges to the Brownian excursion, independently from the offspring distribution (it depends only on the variance of $\xi$ ). The strong "geometrical" relation between a tree and its DFW allows one to have another point of view on the fact that the limit tree-the continuum random tree defined in Aldous (1993) - is the same whatever the distribution of $\xi$. Applying Theorem 1 to lattice distributions, we obtain that in this case also, the limit tree of GaltonWatson trees with lattice distributions is the standard continuum random tree. Indeed, the limit tree is entirely characterized by the limit DFW [see Theorem 20 of Aldous (1993)].

The paper is organized as follows: in Section 2, we present the main results. Section 3 is devoted to the proofs. In 3.1, we exhibit some relations between $V_{n}$ and, in one hand, the depth first queue, and in the other hand, the height process. $V_{n}$ appears then to be closely related to the number of right minima in a discrete excursion; in Section 3.2, we explain how to reduce the study on discrete excursions to the same study on (nonconditioned) random walks. The ends of the proofs are given in Sections 3.3-3.6. Section A.2 is devoted to simulations that illustrate Theorem 2 . 


\section{Results.}

Lattice considerations. $\quad \xi$ may be lattice distributed. In this case, there exists an infinite number of $n$ for which $\Omega_{n}$ is empty. In the sequel of the paper, we note $P P(\xi)$ the set of "possible population sizes" for the trees in $\Omega$, that is,

$$
n \in P P(\xi) \Longleftrightarrow \Omega_{n} \neq \varnothing \text {. }
$$

Convergence spaces. In the sequel of the paper, the five processes described in the Introduction will be considered as continuous processes, with linear interpolation between the integral points. The weak convergence considered in our results is the convergence in $C\left([0,1],\|\cdot\|_{\infty}\right)$.

A first interest of this paper is to give a simple proof of Aldous' theorem:

THEOREM 1. Let $V_{n}$ be the DFW for $\tau \in \Omega_{n}$. For $n \in P P(\xi)$,

$$
\left(\frac{V_{n}(2 n t)}{\sqrt{n}}\right)_{t \in[0,1]} \stackrel{\text { weakly }}{\longrightarrow}\left(\frac{2}{\sigma} e(t)\right)_{t \in[0,1]}
$$

where $(e(t))_{t \in[0,1]}$ is a standard normalized Brownian excursion.

The proof of this theorem relies on the comparison with the DFQP:

THEOREM 2. For any $v>0$, there exist two constants $\gamma>0$ and $N>0$ such that for all $n \geq N$ and $n \in P P(\xi)$,

$$
\mathbb{P}\left(\sup \left\{\left|S_{n}(n t)-\frac{\sigma^{2}}{2} V_{n}(2 n t)\right|, t \in[0,1]\right\} \geq n^{1 / 4+v}\right) \leq e^{-\gamma n^{v}} .
$$

The limit of the DFQP is well known. We state it in the following proposition and we give a short proof.

Proposition 1. For $n \in P P(\xi)$, we have

$$
\left(\frac{S_{n}(n t)}{\sqrt{n} \sigma}\right)_{t \in[0,1]} \stackrel{\text { weakly }}{\longrightarrow}(e(t))_{t \in[0,1]} .
$$

PROOF. Note by $\left(p_{i}\right)_{i \geq 0}$ the distribution of $\xi$, that is, $p_{i}=\mathbb{P}(\xi=i)$. For a tree $\tau$ from $\Omega$, the r.v. $\xi-1$ is $\tilde{p}_{i}=p_{i+1}, \forall i \geq-1$ distributed. By definition (2), $S_{n}$ is the process of sums of the r.v. $\left(\xi_{i}-1\right)$. This is a classical fact for the search queue [see Cormen, Leiserson and Rivest (1990), pages 473-474, or Chassaing and Marckert (2001)] that the queue gets empty only when all the nodes have been visited. The condition $|\tau|=n$ on the tree is then equivalent to the condition

$$
\left\{S_{n}(0)=0, S_{n}(n)=-1, S_{n}(i) \geq 0 \text { for each } i, 1 \leq i \leq n-1\right\} .
$$


Thus, $\left(S_{n}(j)\right)_{0 \leq j \leq n}$ is a discrete excursion. Since the weak convergence of a discrete excursion to a Brownian excursion is well known [Csaki and Mohanty (1981) and Durett, Iglehart and Miller (1977)], the proposition is proved.

Theorem 1 is then a corollary of Theorem 2; note that Theorem 2 shows that, even if $V_{n}$ is not a conditioned random walk (except if $\xi$ is geometrically distributed) it is "close" to the discrete excursion $S_{n}$ for the uniform distance. Some illustrations by simulations are given at the end of the paper.

The height process. At the end of Section 3.1, we will show that the DFW "interpolates" the height process. Theorem 2 and the following Theorem 3 express that $h_{n}$ and $V_{n}$ have very similar properties up to a change of scale with factor 2.

THEOREM 3. For any $v>0$, there exist two constants $\gamma>0$ and $N>0$ such that for all $n \geq N$ and $n \in P P(\xi)$,

$$
\mathbb{P}\left(\sup \left\{\left|S_{n}(n t)-\frac{\sigma^{2}}{2} h_{n}(n t)\right|, t \in[0,1]\right\} \geq n^{1 / 4+v}\right) \leq e^{-\gamma n^{v}} .
$$

COROLlary 1. For $n \in P P(\xi)$, we have

$$
\left(\frac{h_{n}(n t)}{\sqrt{n}}\right)_{t \in[0,1]} \stackrel{\text { weakly }}{\longrightarrow}\left(\frac{2}{\sigma} e(t)\right)_{t \in[0,1]} .
$$

The methods and arguments used in the proofs of Theorems 2 and 3 allow one to derive similar results for other processes associated to the depth first search:

The height of nodes with a given out-degree. Let $h_{n}^{(j)}$ be the process of the height of nodes with out-degree $j$ for $\tau \in \Omega_{n}$ (by convention, the height of a nonexisting node is 0 ).

THEOREM 4. For any $v>0$, there exist two constants $\gamma>0$ and $N>0$ such that for all $n \geq N$ and $n \in P P(\xi)$,

$$
\mathbb{P}\left(\sup \left\{\left|h_{n}(n t)-h_{n}^{(j)}\left(p_{j} n t\right)\right|, t \in[0,1]\right\} \geq n^{1 / 4+v}\right) \leq e^{-\gamma n^{v}} .
$$

COROLlary 2. For $n \in P P(\xi)$, we have

$$
\left(\frac{h_{n}^{(j)}\left(p_{j} n t\right)}{\sqrt{n}}\right)_{t \in[0,1]} \stackrel{\text { weakly }}{\longrightarrow}\left(\frac{2}{\sigma} e(t)\right)_{t \in[0,1]}
$$

This theorem reveals the "good repartition" of the $j$-nodes among all the nodes. It also extends the results obtained by Gutjahr and Pflug (1992) and Marckert (2000) about the height process of the leaves of binary trees (on geometric trees). 
In his paper, Drmota (1994) gives the law of the height of the $i$ th leaf; this can also be obtained by Corollary 2 (with $j=0$ ) using the density of the Brownian excursion [Durett, Iglehart and Miller (1977)]:

$$
\mathbb{P}(e(t) \in d y)=\frac{2 y^{2} \exp \left(-y^{2} / 2 t(1-t)\right)}{\left(2 \pi t^{3}(1-t)^{3}\right)^{1 / 2}} d y .
$$

Occurrences of a given subtree. Note $u$ a fixed tree with size $\lambda$ in $\Omega$ such that

$$
q_{u}=\mathbb{P}(u)>0 .
$$

For $\tau \in \Omega_{n}$, let $h_{n}^{u}$ be the process of the height of nodes that are root of a subtree equal to $u$. We have:

THEOREM 5. For any $v>0$, there exist two constants $\gamma>0$ and $N>0$ such that for all $n \geq N$ and $n \in P P(\xi)$,

$$
\mathbb{P}\left(\sup \left\{\left|h_{n}(n t)-h_{n}^{u}\left(q_{u} n t\right)\right|, t \in[0,1]\right\} \geq n^{1 / 4+v}\right) \leq e^{-\gamma n^{v}}
$$

COROLlary 3. For $n \in P P(\xi)$, we have

$$
\left(\frac{h_{n}^{u}\left(q_{u} n t\right)}{\sqrt{n}}\right)_{t \in[0,1]} \stackrel{\text { weakly }}{\longrightarrow}\left(\frac{2}{\sigma} e(t)\right)_{t \in[0,1]} .
$$

Once again, Theorem 5 reveals the good repartition of the subtrees with a given type in the trees $\tau \in \Omega_{n}$.

We refer to Flajolet, Gourdon and Martinez (1997) and Steyaert and Flajolet (1983) for the same problematic on random binary search trees (recall that BST are not conditioned Galton-Watson trees).

The proof of Theorem 5 allows one to derive a concentration inequality for the number of subtrees with type $u$ in the trees of $\Omega_{n}$. For a tree $\tau$ from $\Omega_{n}$, we note $N_{u}(n)$ the number of occurrences of $u$ in $\tau$, that is, the number of nodes in $\tau$ root of a subtree equal to $u$.

COROLlaRY 4. For any $v>0$, there exist two constants $\gamma>0$ and $N>0$ such that, for all $n \geq N$ and $n \in P P(\xi)$,

$$
\mathbb{P}\left(\left|N_{u}(n)-q_{u} n\right| \geq n^{1 / 2+v}\right) \leq e^{-\gamma n^{v}} .
$$

Conclusion: All the depth first processes have the same limit. Note $D F_{n}$ the process indexed by $[0,1]^{5}$ :

$$
D F_{n}\left(t_{1}, t_{2}, t_{3}, t_{4}, t_{5}\right)=\left(\frac{S_{n}\left(n t_{1}\right)}{\sigma \sqrt{n}}, \frac{V_{n}\left(2 n t_{2}\right)}{\sqrt{n}}, \frac{h_{n}\left(n t_{3}\right)}{\sqrt{n}}, \frac{h_{n}^{(j)}\left(p_{j} n t_{4}\right)}{\sqrt{n}}, \frac{h_{n}^{u}\left(q_{u} n t_{5}\right)}{\sqrt{n}}\right) .
$$

As a consequence of the four last theorems, we have the convergence of the five depth first processes to the same Brownian excursion:

$$
D F_{n}\left(t_{1}, t_{2}, t_{3}, t_{4}, t_{5}\right)_{[0,1]^{5}} \stackrel{\text { weakly }}{\longrightarrow}\left(e\left(t_{1}\right), \frac{2}{\sigma} e\left(t_{2}\right), \frac{2}{\sigma} e\left(t_{3}\right), \frac{2}{\sigma} e\left(t_{4}\right), \frac{2}{\sigma} e\left(t_{5}\right)\right)_{[0,1]^{5}} .
$$




\section{Proofs.}

3.1. Relation between the three depth processes $D F W, D F Q P$ and $h_{n}$. The relations proved in the present subsection are also noted in Le Gall and Le Jan (1998), Le Gall (2000) and Duquesne and Le Gall (2002).

Relation between $S_{n}$ and the height process.

Lemma 1. For any $l \in\{0, \ldots, n-1\}$,

$$
\begin{aligned}
h_{n}(l) & =\sum_{j=1}^{l} I_{\left\{\min _{0 \leq k \leq l-j}\left\{S_{n}(j+k)-S_{n}(j-1)\right\} \geq 0\right\}} \\
& =\#\left\{j \mid 0 \leq j \leq l-1, \min _{0 \leq k \leq l-j}\left\{S_{n}(j+k)\right\}=S_{n}(j)\right\} .
\end{aligned}
$$

The symbol $h_{n}(l)$ denotes then the number of "right" minima (in the weak sense) of $\left(S_{n}(i)\right)_{i=0, \ldots, l}$ on the interval $\llbracket 0, l-1 \rrbracket$.

Proof Of Lemma 1. By definition $h_{n}(l)$ is the depth of the node $v_{l}$ and then it is the number of ancestors of $v_{l}$. Our proof is based on a queue interpretation. We consider a queue with one server where the service rule is: last in, first out; the server serves one client per unit time. Now, consider a tree $t \in \Omega_{n}$ with nodes $\left(v_{0}, \ldots, v_{n-1}\right)$ (sorted according to their first visit time during the depth first procedure). The nodes are the clients. The root, $v_{0}$, arrives at time 0 . The sons of $v_{i}$ arrive at time $i+1$ from the right-most one, to the left-most one (the leftmost one arrives the last, so it will be served the first). At time $i$, the client which is at the head of the queue gets its service and disappears from the queue at time $i+1$.

The queue size in function of time is $\left(S_{n}(i)+1\right)_{i=0, \ldots, n}$, where $S_{n}$ is the DFQP; indeed, when the client $i$ is served $(-1)$, it is replaced by its offspring $\left(+\xi_{i}\right)$, and the queue size at time 0 is 1 .

As an illustration, consider in Figure 2 the node $v_{5}$. It is served at time 5 (which is natural, by construction). In the tree, its height is three since he has three
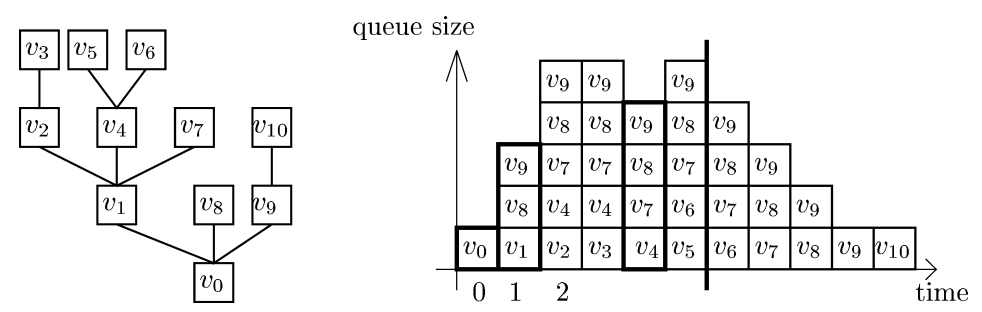

FIG. 2. The LIFO queue associated to a tree. 
ancestors $v_{4}, v_{1}$ and $v_{0}$. The times when its ancestors are served are the times of right minima before time $v_{5}$.

Let us come back to the proof. First, note that by the queue procedure, the parents are served before the children. Consider $v_{k}$. By construction, $v_{k}$ is served at time $k$. Note $t\left(v_{k}\right)$ the subtree of $t$ with root $v_{k}$. After $v_{k}$, the next $\left|t\left(v_{k}\right)\right|-1$ clients that are served are exactly the nodes of $t\left(v_{k}\right)$; but these nodes were not in the queue at time $k$. So, $S_{n}(k) \leq S_{n}(k+i)$, for all $i \in\left\{1, \ldots,\left|t\left(v_{k}\right)\right|-1\right\}$. Moreover, $S_{n}(k)>S_{n}\left(k+\left|t\left(v_{k}\right)\right|\right)$. Indeed, at time $k+\left|t\left(v_{k}\right)\right|$, all the descendents of $v_{k}$ have left the queue. The queue contains the same elements that were in the queue at time $k$ except $v_{k}$ that has disappeared. It follows that $S_{n}(k)$ is a right minimum in the set $\llbracket 0, k+\left|t\left(v_{k}\right)\right|-1 \rrbracket$ but not in $\llbracket 0, k+\left|t\left(v_{k}\right)\right| \rrbracket$.

Consider now $v_{j}$ a node of $t$. All the ancestors of $v_{j}$ are served before $v_{j}$ and are right minima in $\llbracket 0, l-1 \rrbracket$. Moreover, a node $v_{i}$ that is served before time $l$ and that is not an ancestor of $v_{l}$ is not a right minima in $\llbracket 0, l-1 \rrbracket$ since $S_{n}\left(i+\left|t\left(v_{i}\right)\right|\right)<S_{n}(i)$ and $i+\left|t\left(v_{i}\right)\right|<l$ [because in this case $v_{l}$ is not in $t\left(v_{i}\right)$ and all the nodes of $t\left(v_{i}\right)$ are served before $v_{l}$; thus $\left.l>i+\left|t\left(v_{i}\right)\right|\right]$. Thus, the number of right minima of $S_{n}$ in $\llbracket 0, l-1 \rrbracket$ is exactly the number of ancestors of $v_{l}$.

Relation between $V_{n}$ and the height process $h_{n}$. Consider again $\left(v_{0}, \ldots, v_{n-1}\right)$ the set of nodes sorted as said above. For any $l \in\{0, \ldots, n-1\}$ we note

$$
m(l)=\inf \left\{k \mid \tilde{f}(k)=v_{l}\right\}
$$

where $\tilde{f}$ is defined at the beginning of the paper. Since $h_{n}(l)$ is the height of $v_{l}$ for any $l \in\{0, \ldots, n-1\}$, the point $\left(m(l), h_{n}(l)\right)$ belongs to the graph of the DFW $\left\{\left(k, V_{n}(k)\right), k \in\{0, \ldots, 2 n-2\}\right\}$.

LEMMA 2. For any $l \in\{0, \ldots, n-1\}$,

$$
m(l)+h_{n}(l)=2 l \text {. }
$$

ProOF. Consider $h_{n}(l)$ and $h_{n}(l+1)$ the successive heights of the nodes $v_{l}$ and $v_{l+1}$. Two cases arise:

(i) $h_{n}(l+1)=h_{n}(l)+1$. In this case, $v_{l+1}$ is necessarily the left most son of $v_{l}$. And then $m(l+1)=m(l)+1$. Thus $h_{n}(l+1)+m(l+1)=2+h_{n}(l)+m(l)$.

(ii) $h_{n}(l+1) \leq h_{n}(l)$. In this case, the function $\tilde{f}$ visits the ancestors of the node $v_{l}$ till it finds one node $v$ with at least one nonvisited son; $v_{l+1}$ is the left most one of these nonvisited sons. We have

$$
\begin{aligned}
h_{n}(l+1) & =d(\text { root }, v)+1, \\
m(l+1) & =m(l)+h_{n}(l)-d(\text { root }, v)+1 .
\end{aligned}
$$

Thus $h_{n}(l+1)+m(l+1)=m(l)+h_{n}(l)+2$. 
LEMMA 3. For $l \in\{0, \ldots, n-1\}$ and for any $k \in \llbracket m(l), m(l+1) \llbracket$,

$$
\begin{aligned}
V_{n}(m(l+1))-1 & \leq V_{n}(k) \leq V_{n}(m(l)), \\
h_{n}(l+1)-1 & \leq V_{n}(k) \leq h_{n}(l) .
\end{aligned}
$$

PROOF. The DFW interpolates in the following sense the points $\left(m(l), h_{n}(l)\right)$ :

$$
V_{n}(k)= \begin{cases}h_{n}(l), & \text { if } k=m(l) \text { for a given } l, \\ h_{n}(l)-(k-m(l)), & \text { if } k \in \llbracket m(l)+1, m(l+1)-1 \rrbracket \text { for a given } l .\end{cases}
$$

The end of the proof relies on the properties of the depth first search and follows the same arguments as those used in Lemma 2.

3.2. Study via nonconditioned walks, lattice considerations. Consider a random walk $\omega=\left(Z_{i}(\omega)\right)_{i \geq 0}$ with increments $\left(z_{i}\right)_{i \geq 0}$, i.i.d. such that $Z_{0}=0$, $\mathbb{E}\left(z_{1}\right)=0$ and $0<\operatorname{Var}\left(z_{1}\right)<+\infty$. Assume, moreover, that $z_{i}$ takes its values on the set $\{-1,0,1,2, \ldots\}$.

We denote by $R W_{Z}(n)$ the set of $n$ length random walks $\left(Z_{i}\right)_{0 \leq i \leq n}$ with the law induced by the law of the $z_{i}$ and $E X_{Z}(n)$ the set of corresponding "excursions":

$$
\left\{\omega \in E X_{Z}(n)\right\} \Leftrightarrow\left\{\omega \in R W_{Z}(n), Z_{n}(\omega)=-1, Z_{i}(\omega) \geq 0 \forall i \in\{0, \ldots, n-1\}\right\} .
$$

The terminology "excursion" for this kind of path is justified by the fact that adding 1 to each $Z_{i}$ (for $i=1, \ldots n$ ) provides an usual discrete excursion.

Existence and probability of size $n$ excursions (lattice considerations). Let $T_{-1}$ denote the hitting time of -1 by $\left(Z_{i}\right)_{i \geq 0}$ :

$$
T_{-1}=\inf \left\{i \geq 0, Z_{i}=-1\right\} .
$$

We have [Otter's (1949) formula]

$$
\mathbb{P}\left(\omega \in E X_{Z}(n)\right)=\mathbb{P}\left(T_{-1}(\omega)=n\right)=\frac{1}{n} \mathbb{P}\left(Z_{n}=-1\right) .
$$

Since $z_{i}$ may have a lattice distribution, the above probability may be zero for some $n$. Assume that $z_{i}$ takes its values on the lattice $-1+k h$, where $h$ is the maximal span. Let $(a(n))_{n}$ be a sequence of indices such that $a(n) \rightarrow_{n}+\infty$ and $\mathbb{P}\left(Z_{a(n)}=-1\right) \neq 0$. The local central limit theorem [see Port (1994), page 706] says

$$
\frac{\sqrt{a(n)}}{h} \mathbb{P}\left(Z_{a(n)}=-1\right)-\frac{1}{\sqrt{2 \pi} \sigma} \exp \left(-\frac{(-1)^{2}}{2 \operatorname{Var}\left(z_{1}\right) a(n)}\right) \rightarrow 0 \quad \text { as } n \rightarrow+\infty,
$$

and then

$$
\mathbb{P}\left(Z_{a(n)}=-1\right) \sim \frac{h}{\sqrt{2 \pi a(n)} \sigma} .
$$


Hence, for such indices $a(n)$, we have

$$
\mathbb{P}\left(E X_{Z}(a(n))\right) \sim \frac{h}{\sqrt{2 \pi} \sigma a(n)^{3 / 2}} .
$$

When $\xi$ is lattice distributed, the total progeny in the GW process with offspring $\xi$ is also lattice distributed and then the length of the DFW and the length of the DFQP are also lattice distributed. The appearance of the set of indices $a(n)$ is necessary as well as the one of $P P(\xi)$.

From nonconditioned walk to excursion (the conditioning argument). Let $A_{n}$ be a subset of $R W_{Z}(n)$ and $n$ such that $\mathbb{P}\left(Z_{n}=-1\right) \neq 0$. Then

$$
\mathbb{P}\left(A_{n} \mid E X_{Z}(n)\right) \leq \frac{\mathbb{P}\left(A_{n}\right)}{\mathbb{P}\left(E X_{Z}(n)\right)} .
$$

Thus,

$$
\mathbb{P}\left(A_{n}\right)=o\left(n^{-\beta}\right) \Longrightarrow \mathbb{P}\left(A_{n} \mid E X_{Z}(n)\right)=o\left(n^{-\beta+3 / 2}\right) .
$$

This implies that if there exists a moderate deviations principle (or large deviations principle) for a functional of a centered random walk, there exists an upper bound for the analogous principle on the associated excursion. This remark is one of the key points of the present paper and also of Marckert (2000). Its interest is that almost all random variables are much more difficult to handle on $E X_{Z}(n)$ than on $R W_{Z}(n)$. But, exponential concentration type inequalities on $R W_{Z}(n)$ remain true on $E X_{Z}(n)$.

3.3. The number of right minima. The aim of this section is to show a "strong relation" between the number of right minima of a random walk $(W(i))_{0 \leq i \leq l}$ in the set $\llbracket 0, l-1 \rrbracket$ and the value $W(l)-\min _{0 \leq i \leq l}\{W(i)\}$. Thanks to the conditioning argument (Section 3.2), this strong relation will be transported to the excursion in Section 3.4.

Set $l$ a fixed integer and $(W(j))_{j \in\{0, \ldots, l\}}$ a random walk with i.i.d. increments with law $\left(\tilde{p}_{i}\right)_{i \geq-1}$. The number of right minima of $(W(i))_{i}$ [say $\left.R M(l)\right]$ on $\llbracket 0, l \rrbracket$ is:

$$
R M(l)=\#\left\{j \mid 0 \leq j \leq l-1, \min _{0 \leq k \leq l-j}\{W(j+k)\}=W(j)\right\} .
$$

3.3.1. The right minima viewed as ladder epochs on an associated random walk. In order to study the random variable $R M(l)$, we introduce the associated random walk $\left(W^{\bullet}(j)\right)_{j \in\{0, \ldots, l\}}$ defined by

$$
W^{\bullet}(j)=W(l)-W(l-j) \quad \text { for any } j \in\{0, \ldots, l\} .
$$

"Geometrically," the graph of the random walk $W^{\bullet}$ is the reflexion of the graph of $W$ according to the center $(l / 2, W(l) / 2)$. The increments of $\left(W^{\bullet}(i)\right)_{i \geq 0}$ are 
also $\left(\tilde{p}_{i}\right)_{i \geq-1}$ distributed. Denote by $R^{\bullet}(l)$ the number of records (in the large sense) of the sample path $\left(W^{\bullet}(j)\right)_{j=0, \ldots, l}$ :

$$
R^{\bullet}(l)=\#\left\{j, 1 \leq j \leq l, \max _{0 \leq k \leq j}\left\{W^{\bullet}(k)\right\}=W^{\bullet}(j)\right\} .
$$

By (5) we obtain

$$
\begin{aligned}
R M(l) & =R^{\bullet}(l), \\
\max _{0 \leq i \leq l} W^{\bullet}(i) & =W(l)-\min _{0 \leq i \leq l} W(i), \\
\left|\max _{0 \leq j \leq l}\left\{W^{\bullet}(j)\right\}-R^{\bullet}(l) \frac{\sigma^{2}}{2}\right| & =\left|W(l)-\min _{0 \leq i \leq l}\{W(i)\}-R M(l) \frac{\sigma^{2}}{2}\right| .
\end{aligned}
$$

Even if $R^{\bullet}(l)$ and $R M(l)$ are very similar functionals of the paths, $R^{\bullet}(l)$ appears to be easier to handle. Indeed, the record times are stopping times and then, Markov properties of the paths can be used to study $R^{\bullet}(l)$. This is the reason for the construction of the path $W^{\bullet}$.

3.3.2. Concentration inequality on $\max _{0 \leq j \leq l}\left\{W^{\bullet}(j)\right\}$. Our aim here is to show a concentration inequality for the difference between $\frac{\sigma^{2}}{2} R^{\bullet}(l)$ and $\max _{0 \leq j \leq l}\left\{W^{\bullet}(j)\right\}$ :

PROPOSITION 2. For any $v>0$ there exist two constants $\gamma>0$ and $N>0$ such that for any $n \geq N$ and any $l, 0 \leq l \leq n$,

$$
\mathbb{P}\left(\left|\max _{0 \leq j \leq l}\left\{W^{\bullet}(j)\right\}-\frac{\sigma^{2}}{2} R^{\bullet}(l)\right| \geq n^{1 / 4+v}\right) \leq e^{-\gamma n^{v}} .
$$

We can assume that $W^{\bullet}$ is an infinite length r.w. with increment law $\left(\tilde{p}_{i}\right)_{i \geq-1}$. Consider $0=\tau(0)<\tau(1)<\cdots<\tau\left(R^{\bullet}(l)\right)<\cdots$ the weak ladder epochs for $W^{\bullet}$ :

$$
\tau_{k}=\inf \left\{j>\tau_{k-1}, W^{\bullet}(j) \geq W^{\bullet}\left(\tau_{k-1}\right)\right\} \quad \text { for } k \geq 1 .
$$

$R^{\bullet}(l)$ is the number of positive ladder epochs before time $l$. The weak ladder heights are the variables

$$
L H_{k}=W^{\bullet}\left(\tau_{k}\right)-W^{\bullet}\left(\tau_{k-1}\right) \quad \text { for } k \geq 1 .
$$

We have

$$
\max _{0 \leq j \leq l}\left\{W^{\bullet}(j)\right\}=\sum_{k=1}^{R^{\bullet}(l)} L H(k) .
$$

To prove Proposition 2, we need the following lemma. 
LEMMA 4.

$$
\mathbb{E}\left(L H_{1}\right)=\frac{\sigma^{2}}{2}
$$

PROOF. For this proof or more general questions about ladder heights, we refer to Feller [(1971), Chapter XII and pages 425-426]. The fundamental relation is:

$$
\begin{aligned}
& \text { for any } r \geq 0, \\
& \mathbb{P}\left(L H_{1}=r\right)=\mathbb{P}\left(W^{\bullet}(1)=r\right)+\frac{\mathbb{P}\left(W^{\bullet}(1)=-1\right)}{1-\mathbb{P}\left(L H_{1}=0\right)} \mathbb{P}\left(L H_{1}=r+1\right) .
\end{aligned}
$$

Now write

$$
\sum_{r \geq 0} r \mathbb{P}\left(L H_{1}=r\right)=\sum_{r \geq 0} r\left(\mathbb{P}\left(W^{\bullet}(1)=r\right)+\frac{\mathbb{P}\left(W^{\bullet}(1)=-1\right)}{1-\mathbb{P}\left(L H_{1}=0\right)} \mathbb{P}\left(L H_{1}=r+1\right)\right) .
$$

We obtain

$$
\mathbb{E}\left(L H_{1}\right)\left(1-\frac{\mathbb{P}\left(W^{\bullet}(1)=-1\right)}{1-\mathbb{P}\left(L H_{1}=0\right)}\right)=0 .
$$

Using that $\mathbb{E}\left(L H_{1}\right)>0$, we obtain $\mathbb{P}\left(L H_{1}=0\right)=1-\mathbb{P}\left(W^{\bullet}(1)=-1\right)$ and by $(12)$ :

$$
\mathbb{P}\left(L H_{1}=r\right)=\mathbb{P}\left(W^{\bullet}(1)=r\right)+\mathbb{P}\left(L H_{1}=r+1\right)=\mathbb{P}\left(W^{\bullet}(1) \geq r\right) .
$$

The end of the proof is immediate.

REMARK 1. According to (13), $L H_{1}$ has a moment of order $p$ (resp. an exponential moment) if and only if $W^{\bullet}(1)$ has a moment of order $p+1$ (resp. an exponential moment).

ProOF OF Proposition 2. The random walk $W^{\bullet}$ enjoys the strong Markov property, and then, the r.v. $\tau_{k}-\tau_{k-1}$ (for $k \geq 1$ ) are i.i.d. as well as the r.v. $L H_{k}$. Let $K(l, n)$ denote the left-hand side of (9). It follows from (11) that

$$
K(l, n)=\mathbb{P}\left(\left|\sum_{k=1}^{R^{\bullet}(l)}\left(L H_{k}-\frac{\sigma^{2}}{2}\right)\right| \geq n^{1 / 4+v}\right) .
$$

Then

$$
K(l, n) \leq A(l, n)+B(l, n)
$$

where

$$
\begin{aligned}
& A(l, n)=\mathbb{P}\left(R^{\bullet}(l) \geq n^{1 / 2+v}\right) \\
& B(l, n)=\mathbb{P}\left(\left|\sum_{k=1}^{R^{\bullet}(l)}\left(L H_{k}-\frac{\sigma^{2}}{2}\right)\right| \geq n^{1 / 4+v}, R^{\bullet}(l) \leq n^{1 / 2+v}\right)
\end{aligned}
$$


$A(l, n)$ and $B(l, n)$ appears to be probability of moderate deviations of some r.v.; indeed, $R^{\bullet}(l)$, the number of records of a centered random walk is known to be of the order $\sqrt{l}$ and since $\left(\sum_{k=1}^{n}\left(L H_{k}-\frac{\sigma^{2}}{2}\right)\right) / \sqrt{n}$ converges to a Gaussian (nondegenerate) distribution, $B(l, n)$ appears also to be the probability of a rare event. To bound the probability of these rare events, we will use Petrov's results (see the Appendix). We define $\left(U_{k}\right)_{k \geq 0}$ by

$$
U_{k}=\sum_{j=1}^{k}\left(L H_{j}-\frac{\sigma^{2}}{2}\right) .
$$

The r.v. $\left(L H_{j}-\frac{\sigma^{2}}{2}\right)_{j}$ are i.i.d. centered r.v. It follows from Remark 1 and assumptions (1) that there exists a constant $\beta>0$ such that

$$
\mathbb{E}\left(\exp \left\{\beta\left|L H_{i}-\frac{\sigma^{2}}{2}\right|\right\}\right)<+\infty .
$$

Lemmas 5 and 6 allow one to conclude the proof of Proposition 2.

LEMmA 5. There exist two constants $c_{1}>0$ and $N_{1}>0$ such that for any $n \geq N_{1}$ and any $l, 0 \leq l \leq n$,

$$
A(l, n) \leq \exp \left(-c_{1} n^{\nu}\right)
$$

PROOF. In order to simplify notation, in this proof the indices $n^{1 / 2+v}$ have to be read, $\left\lfloor n^{1 / 2+v}\right\rfloor$ :

$$
\begin{aligned}
A(l, n) \leq & \mathbb{P}\left(\tau_{n^{1 / 2+v}} \leq l\right) \\
\leq & \mathbb{P}\left(\tau_{n^{1 / 2+v}} \leq n\right) \\
\leq & \mathbb{P}\left(\max _{0 \leq j \leq \tau_{n^{1 / 2+v}}} W^{\bullet}(j) \leq \max _{0 \leq j \leq n} W^{\bullet}(j)\right) \\
\leq & \mathbb{P}\left(W^{\bullet}\left(\tau_{n^{1 / 2+v}}\right) \leq n^{1 / 2+v / 2}\right)+\mathbb{P}\left(\max _{0 \leq j \leq n} W^{\bullet}(j)>n^{1 / 2+v / 2}\right) \\
\leq & \mathbb{P}\left(U_{n^{1 / 2+v}}+\left\lfloor n^{1 / 2+v}\right\rfloor \frac{\sigma^{2}}{2} \leq n^{1 / 2+v / 2}\right) \\
& +2 \mathbb{P}\left(W^{\bullet}(n) \geq n^{1 / 2+v / 2}-\sqrt{2 \sigma^{2}(n-1)}\right) \\
\leq & \mathbb{P}\left(-U_{n^{1 / 2+v}} \geq\left\lfloor n^{1 / 2+v}\right\rfloor \frac{\sigma^{2}}{2}-n^{1 / 2+v / 2}\right) \\
& +2 \exp \left(-\frac{\left(n^{1 / 2+v / 2}-\sqrt{2 \sigma^{2}(n-1)}\right)^{2}}{2 n g_{w}}\right) \\
\leq & \exp \left(-c_{2} n^{1 / 2+v}\right)+2 \exp \left(-c_{3} n^{v}\right)
\end{aligned}
$$


where $g_{w}$ is the constant $g$ from Petrov lemmas relative to the r.w. $\left(W^{\bullet}(k)\right)_{k \geq 1}$, $c_{2}$ and $c_{3}$ are two constants given in using Petrov's lemmas A2; Lemma A1 has been used to bound the most right term in the fourth inequality.

LEMMA 6. There exist two constants $\tilde{c}_{1}>0$ and $N_{2}>0$ such that for any $n \geq N_{2}$ and any $l, 0 \leq l \leq n$,

$$
B(l, n) \leq \exp \left(-\tilde{c}_{1} n^{\nu}\right)
$$

PROOF.

$$
\begin{aligned}
B(l, n) & \leq \sum_{j=1}^{n^{1 / 2+v}} \mathbb{P}\left(\left|U_{j}\right| \geq n^{1 / 4+v}\right) \\
& \leq \sum_{j=1}^{\left\lfloor c_{4} n^{1 / 4+v}\right\rfloor} \exp \left(-T^{\prime} c_{4} n^{1 / 4+v} / 2\right)+\sum_{j=\left\lfloor c_{4} n^{1 / 4+v}\right\rfloor}^{n^{1 / 2+v}} \exp \left(-\frac{n^{1 / 2+2 v}}{2 j g^{\prime}}\right) \\
& \leq \exp \left(-\tilde{c}_{1} n^{v}\right)
\end{aligned}
$$

for well-chosen constants $\tilde{c}_{1}, g^{\prime}, T^{\prime}, c_{4}$ by Lemma A2, since $L H_{k}$ has exponential moments.

3.3.3. Concentration inequality on $W$. A simple application of Proposition 2 and (8) gives: for any $v>0$ there exist two constants $\gamma>0$ and $N>0$ such that, for any $n, n>N$ and any $l, 0 \leq l \leq n$,

$$
\mathbb{P}\left(\left|W(l)-\min \{W(i), 0 \leq i \leq l\}-R M(l) \frac{\sigma^{2}}{2}\right| \geq n^{1 / 4+v}\right) \leq e^{-\gamma n^{v}} .
$$

One deduces immediately:

COROLlary 5. For any $v>0$ there exist two constants $\gamma>0$ and $N>0$ such that, for any $n, n>N$,

$$
\mathbb{P}\left(\sup _{0 \leq l \leq n}\left\{\left|W(l)-\min \{W(i), 0 \leq i \leq l\}-R M(l) \frac{\sigma^{2}}{2}\right|\right\} \geq n^{1 / 4+v}\right) \leq e^{-\gamma n^{v}}
$$

\subsection{The depth processes.}

Proof of Theorem 3. Consider the excursion $\left(S_{n}(i)\right)_{0 \leq i \leq n}$. We have, for any $l$ smaller than $n$,

$$
\min _{0 \leq i \leq l}\left\{S_{n}(i)\right\}=0
$$


Recall that $S_{n}$ is $\left(W_{i}\right)_{i=0, \ldots, n}$ conditioned to be an excursion. We have

$$
\begin{aligned}
& \mathbb{P}\left(\sup _{0 \leq l \leq n}\left\{\left|S_{n}(l)-h_{n}(l) \frac{\sigma^{2}}{2}\right|\right\} \geq n^{1 / 4+v}\right) \\
& \quad=\mathbb{P}\left(\sup _{0 \leq l \leq n}\left\{\left|W(l)-\min _{0 \leq i \leq l}\{W(i)\}-R M(l) \frac{\sigma^{2}}{2}\right|\right\} \geq n^{1 / 4+v} \mid W \in E X_{W}\right) .
\end{aligned}
$$

It follows from Corollary 5 and (4) that there exists a constant $C>0$ such that

$$
\mathbb{P}\left(\sup _{0 \leq l \leq n}\left\{\left|S_{n}(l)-h_{n}(l) \frac{\sigma^{2}}{2}\right|\right\} \geq n^{1 / 4+v}\right)=O\left(n^{5 / 2}\right) \exp \left(-C n^{v}\right) .
$$

Proof of TheOrem 2. For any $t \in[0,1[$, we denote by $\zeta(n t)$ the integer such that

$$
\begin{gathered}
{[2 n t] \in[m(\zeta(n t)), m(\zeta(n t)+1)[} \\
\mathbb{P}\left(\sup _{t \in[0,1]}\left|S_{n}(n t)-\frac{\sigma^{2}}{2} V_{n}(2 n t)\right| \geq n^{1 / 4+v}\right) \leq A+B+C+D
\end{gathered}
$$

where

$$
\begin{aligned}
A & =\mathbb{P}\left(\sup _{t \in[0,1]}\left|S_{n}(n t)-S_{n}(\zeta(n t))\right| \geq \frac{n^{1 / 4+v}}{3}, \sup _{t}|\zeta(n t)-n t|<n^{1 / 2+v}\right) \\
B & =\mathbb{P}\left(\sup _{t \in[0,1]}\left|S_{n}(\zeta(n t))-\frac{\sigma^{2}}{2} h_{n}(\zeta(n t))\right| \geq \frac{n^{1 / 4+v}}{3}\right) \\
C & =\mathbb{P}\left(\frac{\sigma^{2}}{2} \sup _{t \in[0,1]}\left|h_{n}(\zeta(n t))-V_{n}(2 n t)\right| \geq \frac{n^{1 / 4+v}}{3}\right) \\
D & =\mathbb{P}\left(\sup _{t \in[0,1]}|\zeta(n t)-n t|>n^{1 / 2+v}\right) \\
A & =O\left(n^{3 / 2}\right) \mathbb{P}\left(\sup _{k \leq n^{1 / 2+v}} \sup _{0 \leq j \leq n-k}|W(j)-W(j+k)| \geq \frac{n^{1 / 4+v}}{3}\right) \\
& \leq O\left(n^{3 / 2}\right) \sum_{j=1}^{n} \mathbb{P}\left(\sup _{k \leq n^{1 / 2+v}}|W(k)| \geq \frac{n^{1 / 4+v}}{3}\right) \\
& \leq e^{-c_{5} n^{v}}
\end{aligned}
$$

for a certain constant $c_{5}>0$, if $n$ is large enough using Petrov's lemmas.

According to Theorem 3 , there exists a constant $c_{6}>0$, such that

$$
B=O\left(n^{5 / 2} \exp \left(-c_{6} n^{\nu}\right)\right)
$$


Since $[2 n t] \in[m(\zeta(n t)), m(\zeta(n t)+1)[$, we obtain, by Lemma 3,

$$
\begin{aligned}
C \leq & \mathbb{P}\left(\frac{\sigma^{2}}{2} \sup _{l \in\{1, \ldots, n\}}\left\{\left|h_{n}(l)-h_{n}(l+1)+1\right|\right\} \geq \frac{n^{1 / 4+v}}{3}\right), \\
C \leq & \mathbb{P}\left(\frac{\sigma^{2}}{2} \sup _{l \in\{1, \ldots, n\}}\left\{\left|h_{n}(l+1)-1-\frac{2}{\sigma^{2}} S_{n}(l)\right|\right\} \geq \frac{n^{1 / 4+v}}{6}\right) \\
& +\mathbb{P}\left(\frac{\sigma^{2}}{2} \sup _{l \in\{1, \ldots, n\}}\left\{\left|h_{n}(l)-\frac{2}{\sigma^{2}} S_{n}(l)\right|\right\} \geq \frac{n^{1 / 4+v}}{6}\right) .
\end{aligned}
$$

We conclude using Theorem 3.

To bound $D$, we use (15) and Lemma 2; we have

$$
\begin{aligned}
\sup _{t \in[0,1]}|2 \zeta(n t)-2 n t| & \leq \sup _{t \in[0,1]}|2 \zeta(n t)-m(\zeta(n t))|+\sup _{t \in[0,1]}|m(\zeta(n t))-2 n t| \\
& \leq 2 \sup _{0 \leq l \leq n}\left\{h_{n}(l)\right\}
\end{aligned}
$$

since $h_{n}(l)$ is the number of right minima of $S_{n}$ (see Lemma 3); we use (6) and the conditioning argument to show that

$$
\mathbb{P}\left(\sup _{0 \leq l \leq n}\left\{h_{n}(l)\right\} \geq n^{1 / 2+v}\right)=O\left(n^{3 / 2}\right) \mathbb{P}\left(\sup _{0 \leq l \leq n}\left\{R^{\bullet}(l)\right\} \geq n^{1 / 2+v}\right) .
$$

We conclude using Lemma 5.

\subsection{The heights of nodes with out-degree $j$.}

Proof of TheOrem 4. Consider again the sequence $\left(v_{0}, \ldots, v_{n-1}\right)$ of ordered nodes. Let $\mu(i)$ be the index of the $i$ th node with out-degree $j$, then

$$
h_{n}^{(j)}(i)=h_{n}(\mu(i)) .
$$

The symbol $\mu(i)$ denotes the position of the $i$ th increment $j-1$ in the DFQP, $S_{n}$. Note $\mu^{*}(i)$ the position of the $i$ th increment $j-1$ in $W$ (which is $S_{n}$ with the excursion condition removed); $\mu^{*}(i)$ can be written

$$
\mu^{*}(i)=\sum_{k=1}^{i} g(k)
$$

where the $(g(k))_{k \geq 0}$ are i.i.d. r.v. geometrically distributed with parameter $p_{j}$. Hence, concentration inequality can again be obtained using Petrov's lemmas: For any $v>0$, there exists a constant $C>0$ such that

$$
\mathbb{P}\left(\sup _{0 \leq i \leq n}\left|\mu^{*}(i)-\frac{i}{p_{j}}\right| \geq n^{1 / 2+v}\right)=O\left(\exp \left(-C n^{v}\right)\right) .
$$


Using the conditioning argument (see Section 3.2), we obtain that

$$
\mathbb{P}\left(\sup _{0 \leq i \leq n}\left|\mu(i)-\frac{i}{p_{j}}\right| \geq n^{1 / 2+v}\right)=O\left(n^{3 / 2} \exp \left(-C n^{\nu}\right)\right) .
$$

We conclude using

$$
\begin{aligned}
\sup _{k}\left|h_{n}^{(j)}(k)-h_{n}\left(\frac{k}{p_{j}}\right)\right| \\
\leq \sup _{k}\left|h_{n}(\mu(k))-\frac{2}{\sigma^{2}} S_{n}(\mu(k))\right| \\
\quad+\frac{2}{\sigma^{2}} \sup _{k}\left|S_{n}(\mu(k))-S_{n}\left(\frac{k}{p_{j}}\right)\right| \\
\quad+\sup _{k}\left|\frac{2}{\sigma^{2}} S_{n}\left(\frac{k}{p_{j}}\right)-h_{n}\left(\frac{k}{p_{j}}\right)\right| .
\end{aligned}
$$

The two extreme terms in the right-hand side satisfy concentration exponential inequality by Theorem 3; we carry out exponential bound for the last term using the same computation as that used for $A$ and $D$ in (16) and (17).

REMARK 2. Another object of interest is the number of nodes with outdegree $j$ :

$$
N_{j}^{(n)}=\#\left\{i \mid \xi_{i}=j\right\} .
$$

The exact law of $N_{j}^{(n)}$ seems to be difficult to compute because the tree is conditioned to be of size $n$. But, with our method we can obtain moderate deviations. Indeed, if we remove the tree size condition, the sequence $\left(\xi_{i}\right)_{0 \leq i \leq n-1}$ is a sequence of i.i.d. r.v., $N_{j}^{(n)}$ is binomial $B\left(n, p_{j}\right)$ distributed and then $N_{j}^{(n)}$ is concentrated around $n p_{j}$. Now, using the conditioning argument given in Section 3.2, one finds that for any $v>0$, there exists a constant $C$ such that

$$
\mathbb{P}\left(\left|N_{j}^{(n)}-p_{j} n\right| \geq n^{1 / 2+v}\right)=O\left(\exp \left(-C n^{v}\right)\right) .
$$

Other elements about the law of $N_{j}^{(n)}$ and about the joint law of the $\left(N_{j}^{(n)}\right)_{j}$ are given in Drmota (1996).

3.6. Occurrence of a given subtree. We denote by $N_{u}(n)$ the number of nodes in $\tau \in \Omega_{n}$ roots of a subtree equal to $u$. For $1 \leq i \leq N_{u}(n)$, let $\phi(i)$ denote the index of the $i$ th node among $\left(v_{0}, v_{1}, \ldots, v_{n-1}\right)$, that is the root of a $u$-type subtree and

$$
\rho(i)=\phi(i)+\lambda-1
$$


Each (finite) tree $t$ from $\Omega$ can be described by its sequence of successive progenies (SSP), Seq $=\left(\xi_{0}, \ldots, \xi_{n-1}\right)$, where $n$ is the number of nodes of $t$. We denote by $\operatorname{Seq}(i, j)$ the sequence $\left(\xi_{i}, \ldots, \xi_{i+j-1}\right)$ and by $\operatorname{Seq}(i)$ the $(i+1)$ st coordinate of $S e q$, in order that $\operatorname{Seq}(i)=\xi_{i}$. Let Word $=\left(a_{0}, \ldots, a_{\lambda-1}\right)$ denote the SSP of the subtree $u$. In a first step, we establish a concentration theorem for occurrences of Word in a sequence $\operatorname{Seq}^{*}$, when the $\left(\operatorname{Seq}^{*}(i)\right)_{i}$ are i.i.d. r.v. with the same law as $\xi$. Then, by the connexion given in Section 3.2, we transport the nonconditioned concentration type inequality obtained on $\mathrm{Seq}^{*}$ to a concentration type inequality on $S e q$, that is, on $\Omega_{n}$.

So consider $\left(\operatorname{Seq}^{*}(i)\right)_{i}$ and set $N_{u}^{*}(n)$ the number of occurrences of Word in $S e q^{*}$. For $1 \leq i \leq N_{u}^{*}(n)$, we define the sequence $\left(\phi^{*}(i)\right)_{i}$ by

$$
\begin{aligned}
& \phi^{*}(1)=\inf \left\{k, \operatorname{Seq}^{*}(k, k+\lambda-1)=\text { Word }\right\}, \\
& \phi^{*}(i)=\inf \left\{k, k>\phi^{*}(i-1), \operatorname{Seq}^{*}(k, k+\lambda-1)=\text { Word }\right\} .
\end{aligned}
$$

An important point is that, by the properties of the subtree (and its induced DFW), two sequences Word in Seq* can not overlap. To express more simply the Markov properties of $\left(\phi^{*}(i)\right)_{0 \leq i}$ for $1 \leq i \leq N_{u}^{*}(n)$, we set

$$
\rho^{*}(i)=\phi^{*}(i)+\lambda-1 .
$$

The symbol $\rho^{*}(i)$ is the index of the last letter of the $i$ th occurrence of Word in $S e q^{*}$. We have

$$
\rho^{*}(1)+1 \stackrel{d}{=} \rho^{*}(i+1)-\rho^{*}(i) \quad \text { for all } i \geq 1
$$

[we set $\left.\rho^{*}(0)=-1\right]$.

Mean and tail of the distribution of $\rho^{*}(1)$.

$$
\begin{aligned}
\mathbb{P}\left(\rho^{*}\right. & (1)=\lambda+k-1) \\
& =\mathbb{P}\left(\rho^{*}(1)>k-1 \cap\left(\operatorname{Seq}^{*}(k, \lambda)=\text { Word }\right)\right) \\
& =\mathbb{P}\left(\rho^{*}(1)>k-1\right) \mathbb{P}\left(\operatorname{Seq}^{*}(k, \lambda)=\text { Word }\right) \\
& =\mathbb{P}\left(\rho^{*}(1)>k-1\right) \mathbb{P}\left(\operatorname{Seq}^{*}(0, \lambda-1)=\text { Word }\right) .
\end{aligned}
$$

The first equation comes from the fact that two sequences Word can not overlap. The second is due to the fact that the $\left(\operatorname{Seq}^{*}(i)\right)_{i}$ are independent.

We have

$$
\mathbb{P}\left(\operatorname{Seq}^{*}(0, \lambda-1)=\text { Word }\right) \stackrel{\text { def }}{=} q_{u}=\prod_{i} p_{i}^{n_{i}} \quad \text { where } n_{i}=\#\left\{j \mid a_{j}=i\right\} .
$$

Since $\mathbb{P}\left(\rho^{*}(1)<\lambda-1\right)=0$, the distribution of $\rho^{*}(1)$ is well defined by the above equations.

We sum equality (23) from $k$ equal 0 to $+\infty$; we obtain

$$
1=q_{u}\left(\mathbb{E}\left(\rho^{*}(1)\right)+1\right)
$$


and then

$$
\mathbb{E}\left(\rho^{*}(1)\right)+1=\mathbb{E}\left(\rho^{*}(i+1)-\rho^{*}(i)\right)=q_{u}^{-1} .
$$

Let $k=m \lambda+r$, with $0 \leq r<\lambda$

$$
\left\{\rho^{*}(1)>k\right\} \subset \bigcap_{0 \leq s \leq m-1}\{\operatorname{Seq}(s \lambda, \lambda) \neq \text { Word }\} .
$$

Since the events in the right-hand side are independent and have the same probability,

$$
\begin{aligned}
\mathbb{P}\left(\rho^{*}(1)>k\right) & \leq\left(1-q_{u}\right)^{m} \\
& \leq \beta \delta^{k}
\end{aligned}
$$

where $\delta=\left(1-q_{u}\right)^{1 / \lambda}$, and $\beta=\left(1-q_{u}\right)^{-1}$. Thus $\rho^{*}(1)$ has exponential moment.

Now, one can copy the arguments given in Section 3.5. Indeed, we have

$$
\rho^{*}(j)=1+\sum_{i=1}^{j}\left(\rho^{*}(i)-\rho^{*}(i-1)\right)
$$

to be compared with (19). We have

$$
\mathbb{P}\left(\sup _{0 \leq i \leq N_{u}^{*}(n)}\left|\rho^{*}(i)-\frac{i}{q_{u}}\right| \geq n^{1 / 2+v}\right) \leq \mathbb{P}\left(\sup _{0 \leq i \leq n}\left|r^{*}(i)-\frac{i}{q_{u}}\right| \geq n^{1 / 2+v}\right)
$$

where $\left(r^{*}(i)\right)_{i \geq 0}$ is a random walk with increment distributed as $\rho^{*}(1)$. Using the existence of an exponential moment for $\rho^{*}(1)$ and Petrov's lemmas, we obtain that for any $v>0$, there exists a constant $C>0$ such that

$$
\mathbb{P}\left(\sup _{0 \leq i \leq N_{u}^{*}(n)}\left|\rho^{*}(i)-\frac{i}{q_{u}}\right| \geq n^{1 / 2+v}\right)=O\left(\exp \left(-C n^{v}\right)\right)
$$

and then by the conditioning arguments,

$$
\mathbb{P}\left(\sup _{0 \leq i \leq N_{u}(n)}\left|\rho(i)-\frac{i}{q_{u}}\right| \geq n^{1 / 2+v}\right)=O\left(n^{3 / 2} \exp \left(-C n^{v}\right)\right)
$$

to be compared with (20). The end of the proof is similar to the end of the proof of Proposition 2.

ProOF OF COROllary 4. We have

$$
N_{u}(n)=\max \{i \mid \rho(i) \leq n\} \quad \text { and } \quad N_{u}^{*}(n)=\max \left\{i \mid \rho^{*}(i) \leq n\right\} .
$$

Then

$$
\begin{aligned}
\mathbb{P}\left(\left|N_{u}(n)-q_{u} n\right| \geq n^{1 / 2+v}\right)= & \mathbb{P}\left(\left|N_{u}(n) / q_{u}-n\right| \geq n^{1 / 2+v} / q_{u}\right) \\
\leq & \mathbb{P}\left(\left|N_{u}(n) / q_{u}-\rho\left(N_{u}(n)\right)\right| \geq n^{1 / 2+v} /\left(2 q_{u}\right)\right) \\
& +\mathbb{P}\left(\left|\rho\left(N_{u}(n)\right)-n\right| \geq n^{1 / 2+v} /\left(2 q_{u}\right)\right) .
\end{aligned}
$$


The first term in the right-hand side is exponentially small thanks to (26). For the second term, we have

$$
\begin{aligned}
& \mathbb{P}\left(\left|\rho\left(N_{u}(n)\right)-n\right| \geq n^{1 / 2+v} /\left(2 q_{u}\right)\right) \\
& \quad=O\left(n^{3 / 2}\right) \mathbb{P}\left(\left|\rho^{*}\left(N_{u}^{*}(n)\right)-n\right| \geq n^{1 / 2+v} /\left(2 q_{u}\right)\right) .
\end{aligned}
$$

Since

$$
\left|\rho^{*}\left(N_{u}^{*}(n)\right)-n\right| \leq \rho^{*}\left(N_{u}^{*}(n)+1\right)-\rho^{*}\left(N_{u}^{*}(n)\right)
$$

and by the Markov property $\rho^{*}\left(N_{u}^{*}(n)+1\right)-\rho^{*}\left(N_{u}^{*}(n)\right)={ }^{d} \rho^{*}(1)+1$, we have

$$
\begin{aligned}
\mathbb{P}\left(\left|\rho\left(N_{u}(n)\right)-n\right| \geq n^{1 / 2+v} /\left(2 q_{u}\right)\right) \\
\quad \leq O\left(n^{3 / 2}\right) \mathbb{P}\left(\rho_{1}^{*} \geq n^{1 / 2+v} /\left(2 q_{u}\right)-1\right) \\
\quad \leq \beta \delta^{n^{1 / 2+v} /\left(2 q_{u}\right)} .
\end{aligned}
$$

This concludes the proof of the corollary.

\section{APPENDIX}

A.1. Petrov's results about moderate deviations of random walk. The two following lemmas are direct corollaries of Theorems 2.2, 2.4 and 2.6 from Petrov (1975).

LEMMA A1. Let $\left(Z_{i}\right)_{i \geq 0}$ a random walk with increment $z_{i}$, centered, with $\mathbb{E}\left(z_{i}^{2}\right)<+\infty$. Then, for every $x$,

$$
\mathbb{P}\left(\max _{0 \leq k \leq n}\left\{Z_{k}\right\} \geq x\right) \leq 2 \mathbb{P}\left(Z_{n} \geq x-\sqrt{2(n-1) \mathbb{E}\left(z_{i}^{2}\right)}\right) .
$$

LEMMA A2. If, moreover, there exists a constant $a>0$ such that $\mathbb{E}\left(e^{a\left|z_{1}\right|}\right)<$ $+\infty$ then there exist a constant $g>0$ and a constant $T>0$ such that

$$
\begin{array}{ll}
\mathbb{P}\left(Z_{n} \geq x\right) \leq \exp \left(-\frac{x^{2}}{2 n g}\right) & \text { if } 0 \leq x \leq n g T, \\
\mathbb{P}\left(Z_{n} \geq x\right) \leq \exp \left(-\frac{T x}{2}\right) & \text { if } x \geq n g T .
\end{array}
$$

A.2. Simulations. Theorem 2 possesses very visual interpretations, as can be seen on the following simulations.

1. In the first figure we have simulated a tree with size 5560 and with lattice offspring distribution $p_{0}=13 / 18, p_{2}=1 / 6, p_{6}=1 / 9$; the corresponding value of $\sigma^{2} / 2$ is $11 / 6$. In black, the DFW, in grey the DFQP. 

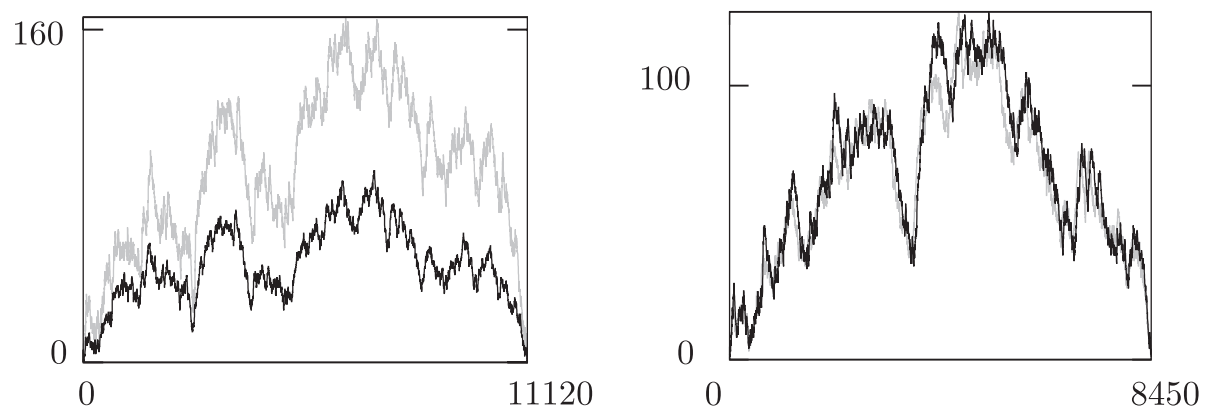

One can observe the quasi proportionality of the two processes. Note also that since $2 l \geq m(l)$ (for any $l$ ), the DFW "lags" from the DFQP.

2. In the second figure above we have simulated a size 4208 tree with a nonlattice offspring distribution $p_{0}=8 / 15, p_{1}=4 / 15, p_{3}=2 / 15, p_{5}=1 / 15$ (in this case $\left.\sigma^{2} / 2=16 / 15\right)$.

A.3. Conclusion. We have obtained exponential bounds for the probability of small deviations for the uniform distance between the normalized depth processes, that is, for example,

$$
\mathbb{P}\left(\sup _{0 \leq t \leq 1}\left|\frac{S_{n}(2 n t)}{\sqrt{n}}-\frac{\sigma^{2} h_{n}(n t)}{2 \sqrt{n}}\right| \geq n^{-1 / 4+v}\right) \leq e^{-\gamma n^{\nu}} .
$$

A corollary of these results is that the five depth processes have the same Brownian excursion as limit.

If one only wants to obtain the weak convergence of the five processes to the same limit, one may weaken the assumption on $\xi$ and only assumes the existence of moment of order $p$ (instead of the existence of an exponential moment). In this case, Petrov's inequality may be replaced by the Fuk and Nagaev (1971) inequality [see also Petrov (1975), page 78]

$$
\mathbb{P}\left(W_{n} \geq x\right) \leq \frac{c_{1} n}{x^{p}}+\exp \left(-c_{2} \frac{x^{2}}{n}\right),
$$

for two constants $c_{1}>0$ and $c_{2}>0$.

Taking $p \geq 9$ and using the same proofs as the ones given in the present paper, one shows that the uniform distances between the normalized depth processes converge to 0 in probability.

One can probably obtain this convergence for smaller values of $p$ with much more technical computations, but this is not in the spirit of the present paper.

One can note that the ladder height has a finite variance if and only if $p \geq 3$, and so, we can conjecture that in this case the uniform distance between $S_{n}$ and $V_{n}$ converges to zero; some simulations "show" that this convergence to zero is not "obvious" if $p<3$. 


\section{REFERENCES}

Aldous, D. (1991). The continuum random tree. II. An overview. In Stochastic Analysis (M. T. Barlow and N. H. Bingham, eds.) 23-70. Cambridge Univ. Press.

Aldous, D. (1993). The continuum random tree. III. Ann. Probab. 21 248-289.

Aldous, D. (1998). Brownian excursion conditioned on its local time. Elect. Comm. Probab. 3 79-90.

Chassaing, P., Marckert, J. F. and Yor, M. (2000). The height and width of simple trees. In Mathematics and Computer Science: Algorithms, Trees, Combinatorics and Probabilities (D. Gardy and A. Mokkadem, eds.) 17-30. Birkhäuser, Boston.

Chassaing, P. and MARCKert, J. F. (2001). Parking functions, empirical processes, and the width of rooted labeled trees. Elec. J. Combin. 8 R14.

Cormen, T. H., Leiserson, C. E. and Rivest, R. L. (1990). Introduction to Algorithms. MIT Press.

CSAKI, E. and Mohanty, S. G. (1981). Excursion and meander in random walk. Canad. J. Statist. $957-70$.

Drmota, M. (1994). The height distribution of leaves in rooted trees. Discrete Math. Appl. 4 45-58.

Drmota, M. (1996). On nodes of given degree in random trees. In Probabilistic Methods in Discrete Mathematics (V. F. Kolchin, V. Ya. Kozlov, V. V. Mazalov, Yu. L. Pavlov and Yu. V. Prokhorov, eds.). VSP, Zeist, The Netherlands.

Drmota, M. and GitTEnberger, B. (1997). On the profile of random trees. Random Structures Algorithms 10 421-451.

Duquesne, T. and Le Gall, J. F. (2002). Random Trees, Lévy Processes and Spatial Branching Processes. Astérisque 281.

Durrett, R. T., Iglehart, R. D. L. and Miller, D. R. (1977). Weak convergence to Brownian meander and Brownian excursion. Ann. Probab. 5 117-129.

Feller, W. (1971). An Introduction to Probability Theory and Its Applications 2, 2nd ed. Wiley, New York.

Flajolet, P., Gourdon, X. and Martinez, C. (1997). Patterns in random binary search trees. Random Structures Algorithms 11 223-244.

Fuk, D. H. and Nagaev, S. V. (1971). Probability inequalities for sums of independent random variables. Theory Probab. Appl. 16 643-660.

Gutjahr, W. and Pflug, G. C. (1992). The asymptotic contour process of a binary tree is a Brownian excursion. Stochastic Process. Appl. 41 69-89.

Kennedy, D. P. (1976). The distribution of the maximum Brownian excursion. J. Appl. Probab. 13 371-376.

LE GALL, J. F. (2000). Lévy processes and branching processes. Courses Notes.

LE GALL, J. F. and LE JAN, Y. (1998). Branching processes in Lévy processes: The exploration process. Ann. Probab. 26 213-252.

LIMIC, V. (2000). On the behavior of LIFO preemptive resume queues in heavy traffic. Electron. Comm. Probab. 5 13-27.

Limic, V. (2001). A LIFO queue in heavy traffic. Ann. Appl. Probab. 11 301-331.

MARCKERT, J. F. (2000). The contour of size $n$ general planar trees. Preprint 53-00, Univ. Versailles.

Meir, A. and Moon, J. W. (1978). On the altitude of nodes in random trees. Canad. J. Math. 30 997-1015.

Otter, R. (1949). The multiplicative process. Ann. Math. Statist. 20 206-224.

Petrov, V. V. (1975). Sums of Independant Random Variables. Springer, Berlin.

PORT, S. C. (1994). Theoretical Probability for Applications. Wiley, New York. 
Steyaert, J. M. and Flajolet, P. (1983). Patterns and pattern-matching in trees: An analysis. Inform. Control 58 19-58.

TAKÁCS, L. (1993). Limit distributions for queues and random rooted trees. J. Appl. Math. Stochastic Anal. 6 189-216.

\author{
DÉPARTEMENT DE MATHÉMATIQUES \\ UNIVERSITÉ DE VERS AILLES \\ 45 AVENUE DES ETATS UNIS \\ 78035 Vers Ailles CEDEX \\ FRANCE \\ E-MAIL: $\{$ marckert, mokkadem\}@ math.uvsq.fr
}

